\title{
Saffron Interactive, Create Your Own Future
}

\section{Case Study for the Effective Use of AI Technology within Blended Learning}

https://doi.org/10.3991/ijac.v14i2.25697

\author{
Noorie Sazen $(\bowtie)$ \\ Saffron Interactive, London, United Kingdom \\ info@saffroninteractive.com
}

\begin{abstract}
This is a case study detailing the 2021 IELA Gold award winner for Blended Learning, Saffron Interactive's Create Your Own Future. This project was developed to support those most at risk from digital disruption into the labour market and towards sustainable employment.
\end{abstract}

Keywords - blended learning, artificial intelligence, natural language processing, sentiment analysis, employment support, career adaptability, skills assessment, data analytics

\section{Introduction}

The world has been facing a reskilling crisis as a result of digital disruption, even prior to COVID-19; however, the pandemic has accelerated this issue. In particular, it has affected those who are in more manual jobs and have historically had lower educational attainment, those who have the least access to training from their employers, and those with the least ability to purchase training. Create Your Own Future, Saffron Interactive's employability support platform, was designed to respond to this pressing need: supporting individuals on their personal journey towards sustainable employment, enabling them to harness the valuable transferrable skills already developed and to encourage career adaptability. A solution was required that could be deployed on a mass scale.

\section{The project}

Nesta, an innovation foundation and The Department for Education (UK), chose Saffron as an innovator to develop a digital solution to the lack of career adaptability within this target cohort, and it was to be made available to the general public. Saffron partnered with the Trades Union Congress so that the solution could be deployed to members of unions.

During the pre-development stage, Saffron conducted over 15 focus groups consisting of 60 work coaches who supported individuals or participants of the target 
cohort profile. By combining the findings from these focus groups, alongside designbased thinking principles, it was established that the solution would need to cater for:

- Low literacy skills

- Low digital skills

- Neurodiversity

- Low self-esteem

- Low career aspirations

Alongside this, Saffron needed to consider those individuals with previously bad experiences of education who may be resistant to learning, as well as those who were "time-poor" due to many individuals juggling more than one job to make ends meet.

Large employers that are unionised in the UK tend to have learning representatives within their organisations who are given allocated hours to encourage development within their workforce. We partnered with these union learning representatives, as well as target learners, to design, develop, test, re-iterate, refine, and deploy Create Your Own Future.

\section{Our approach}

In order to respond to this complex challenge, Saffron's considerable experience in creating digital solutions that enable lasting behavioural change was key. To enable and encourage those most vulnerable to take charge of their own future, it was essential for users to see career planning and skills development as attainable and not as a one-time fix, but something they can and should be doing throughout their working lives.

We used the Career Construction theory by Mark Savickas [1], as the basis of our theory of change. We incorporated the user's need to find their vocational personality, fit within their life goals, ambitions, barriers, and motivations to ignite the 4Cs of Career Adaptability - Concern, Control, Curiosity and Confidence. We added "Commitment" from the University of Warwick's Jenny Bimrose and another C-"Capability"from our own experience.

We knew that coaching or mentoring had been shown in many different constructs to yield a better outcome than an individual trying to get to the same place on their own. So we created an AI-enabled video mentor to help individuals determine their goals, ambitions, barriers and meaning in their work. The platform allowed individuals to "talk" to their mentor through their phone, tablet or desktop device using voice recognition. Individuals could also upload video, if they wanted.

As the user continues the conversation with their mentor, they are guided to a skills assessment to "value" their transferrable skills, matching these to potential job roles and the requisite online training that may support their application. Users are then encouraged to "pin" careers and learning they would like to explore and to make actionable goals in the platform. Behavioural nudges, reflection points and light gamification are embedded throughout, to keep users on track, celebrate achievement of milestones and form personal prompts to encourage completion. 


\section{The user journey}

Planning for your future career is an ongoing journey, which is why Create Your Own Future is there as long as the user needs it to be. The platform provides individuals with continual opportunities to re-assess their skills, update their career aspirations and create new goals. The user journey can be broken out into key sections:

\subsection{AI-enabled video mentor}

Each of the four mentors (see Figure 1) were chosen based on comprehensive focus group research, offering a range of ages, ethnicities, personalities, digital experience and career histories. By having a real person to complete their journey with, the learner is given continuous personal guidance and support.

Throughout their journey, the learner has interactive conversations with their mentor to discuss their feelings, aspirations and confidence levels about achieving the future they want. The AI-enabled mentor reacts through sentiment analysis to direct the learner down a particular path, depending on what they've said and how they're feeling. The mentor also directs individuals to make use of "live" human support, where needed.

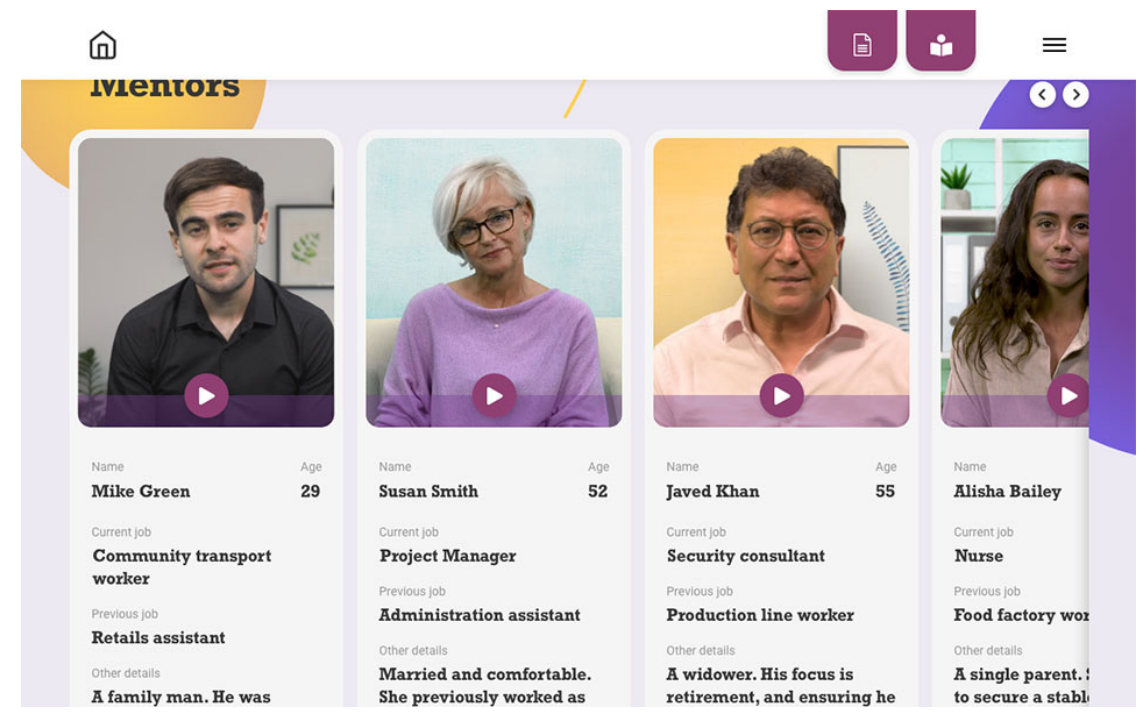

Fig. 1. The learner is offered a choice of four AI mentors

\subsection{Skills assessment and career matching}

The user works through a dynamic card-based skills assessment diagnostic (see Figure 2). This encourages an individual to reflect on their existing transferrable skillset and offers recognition as to the value of the skills and experiences that they have gained throughout life - in the world of work and beyond. 
同

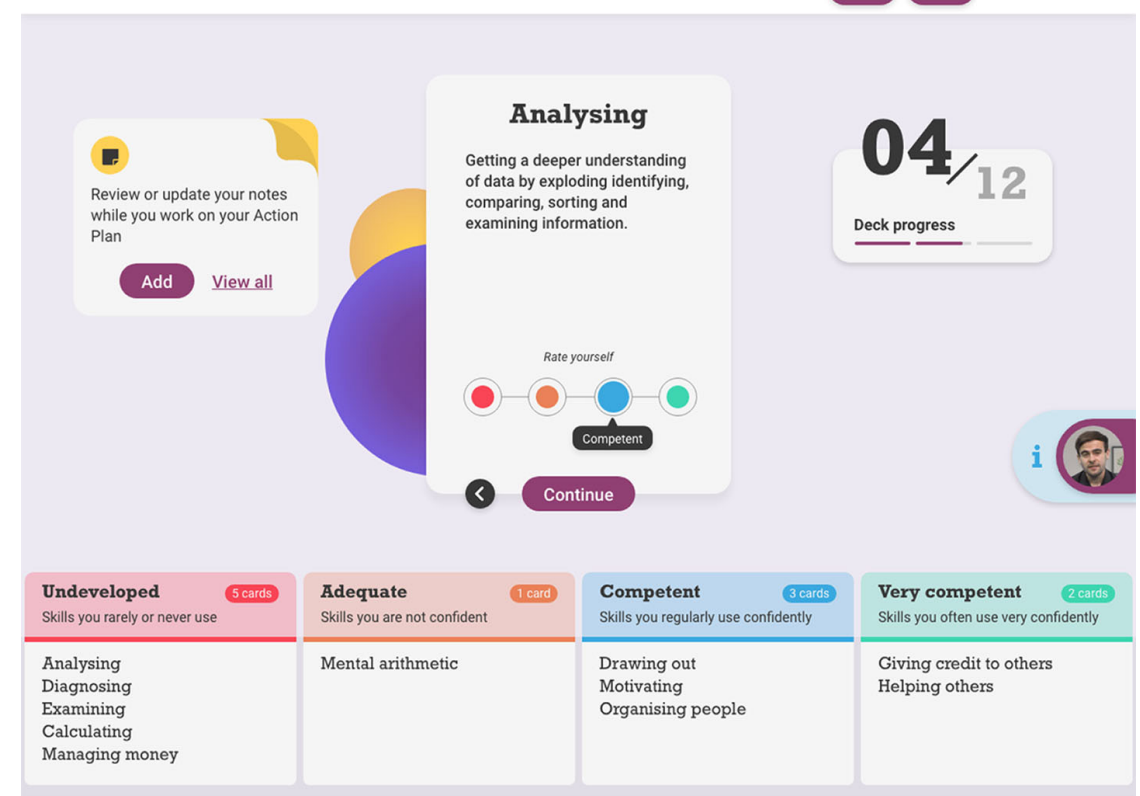

Fig. 2. The learner progresses through 36 transferrable skills cards

The user is then presented with personalised career and training recommendations, categorised into job families (see Figure 3). They are able to quickly see the skills they currently have and those they'll need to develop further. The "pin" functionality allows the learner to save any jobs and free training they're interested in.

同

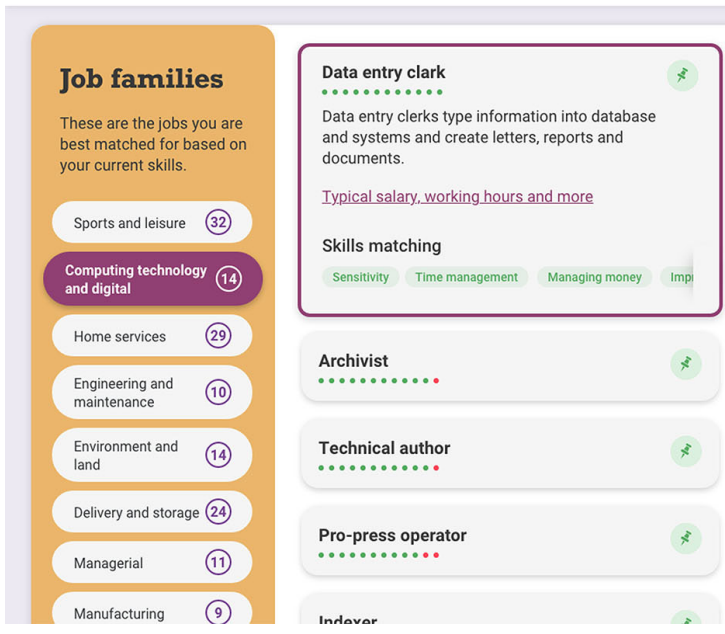

입 을

\section{Training}

These are the jobs you are best matched for based on your current skills.

x

Principles of customer

service

8-12 weeks Level 2

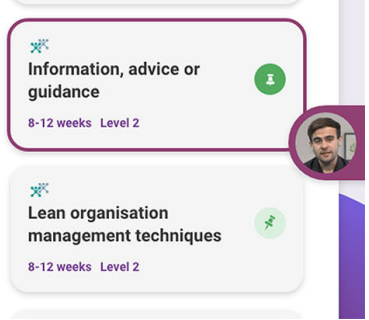

Fig. 3. The learner is presented with personalised career and training results 


\subsection{Action planning and dynamic dashboard}

Create Your Own Future focuses on empowerment and positive behavioural shifts, encouraging learners to take ownership of their career path and make a lasting change. The Action Plan gives the user the opportunity to create an interactive "to-do" list, which they can update and refine as often as they need.

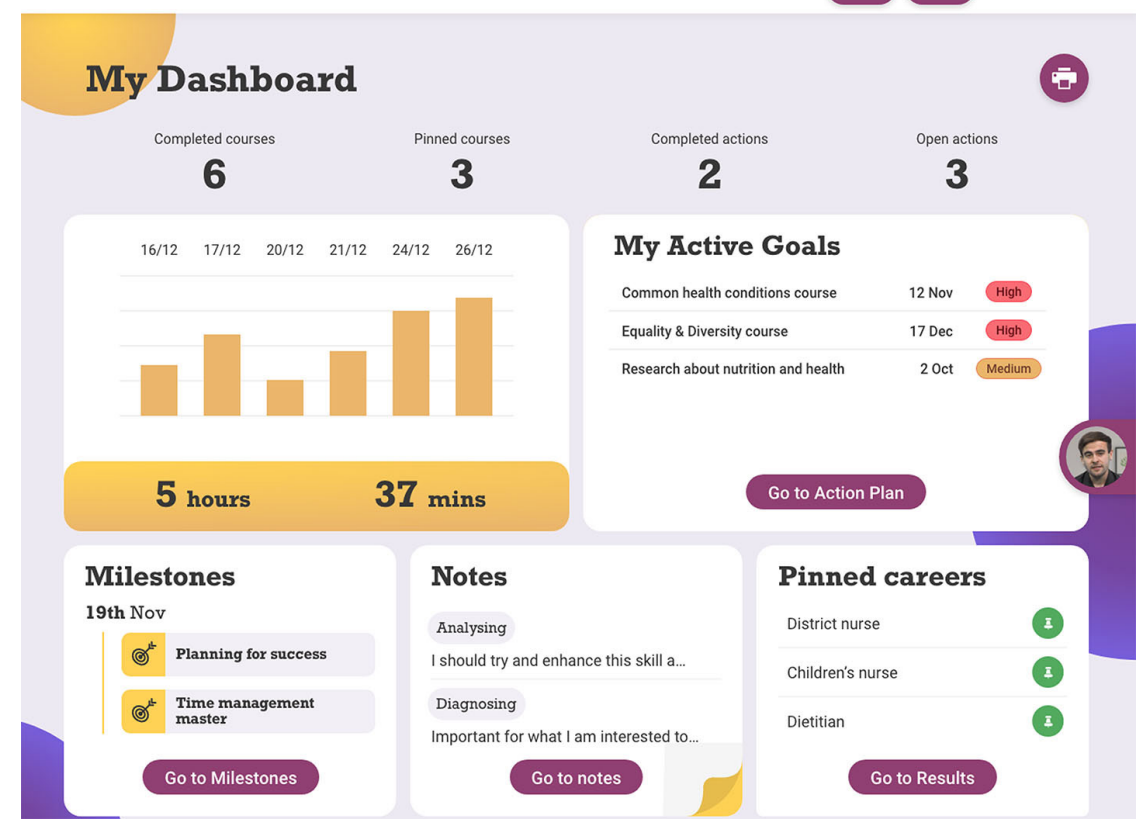

Fig. 4. The learner's continually evolving dynamic dashboard

Culminating the journey is the user's dashboard (see Figure 4). Comprising an interactive learning and development portal that unites all the stages of the user's journey so far. This offers the user an engaging hub where they can return, as their journey towards sustainable employment and becoming a life-long learner continues.

\section{4 'Big data' and reporting}

The Create Your Own Future platform records every decision point and conversation, allowing rich data analytics to be gained. Every user journey is unique but has between 200 to 300 recorded points so that every action or reaction a user has can be analysed. The "back end" data can also be used by human advisers/mentors to see a "real-time" indication of when a user is not engaging and, therefore, needs human interventions. This offers mentors the opportunity to reach out with a call, an email or text, to remotivate the user to complete goals or learning. 


\section{The results}

Despite the challenges faced as a result of the global pandemic, Create Your Own Future's pilot project of just under 500 users spent a combined 51,120 minutes in the platform over 4 months. This enabled Saffron to glean some incredible data insights. In terms of confidence and control, there was a $40 \%$ increase in users who reported feeling confident to take on their future after working through the platform, compared to when they started. Alongside this, over $90 \%$ of users cited that the platform encouraged them to consider careers they had never previously thought about — an indication that Create Your Own Future was able to equip and nurture individuals to take their first steps towards career adaptability.

The platform also received an overwhelmingly positive response in terms of user experience, with $80 \%$ of users feeling that their AI mentor was both supportive and engaging. Most individual features of the platform were also rated highly; $100 \%$ of users cited the skills assessment, action plan and dashboard as very intuitive and easy to use.

As users also completed learning in the platform, the bite-sized modules that supported career adaptability and turned thought into action were also rated highly. Both neuro diverse and other users felt that the experience of using the AI mentor was particularly useful at the beginning of the journey when career aspirations and concerns were being addressed, as well as when career recommendations had been made, with the mentor able to support with sorting through how this information could translate to next steps. This shows that the AI capability and sentiment analysis, alongside the voice-recognition integration, was of a high quality.

\section{$6 \quad$ Reference}

[1] Savickas, M. (2019). Career Construction Theory: Life Portraits of Attachment, Adaptability, and Identity. Mark L. Savickas.

\section{$7 \quad$ Author}

Noorie Sazen is the CEO of Saffron Interactive, 1-7 Pilgrimage St, London SE1 4LL, United Kingdom.

Noorie is a former partner at KPMG, having led their People Services business in London and Eastern Counties. She combines an arsenal of business experience, consultancy and change management skills with a love of learning. This has helped her keep Saffron at the forefront of learning technologies innovation.

A sharp analytical mind helps drive business growth for Saffron's clients. Noorie opens unseen commercial opportunities through strategic change campaigns and learning interventions. A passion for behavioural science informs these, driving transformational performance improvement.

Having worked in both practice and industry, including for BP and Lendlease, Noorie brings a wealth of perspectives to the table. She knows how to manage skills gaps to ensure organisations treat change as a force for good. Email: info@saffroninteractive.com

Article submitted 2021-07-23. Resubmitted 2021-09-19. Final acceptance 2021-09-23. Final version published as submitted by the authors. 